\title{
Visual Observations as Exercises in Physics
}

\author{
Jozef Drga - Martin Bulko - Karol Petrík-Mária \\ Csatáryová - Stanislav Šimkovič ${ }^{*}$
}

\begin{abstract}
Visual meteor observations are a fun and interesting approach to astronomy and to scientific research in general. It can be used for laboratory or practical exercises in physics at high schools and universities. The students can personally collect and analyze the acquired data. The output consists of Zenithal Hourly Rate (ZHR) values, spatial density and population index. In this paper, the so called counting method is described as it is the most suitable method for beginners. As a practical example, the ZHR curve of the Lyrid meteor shower was evaluated and the maximum and the duration of the shower were calculated.
\end{abstract}

Key words: meteors, practical exercises in physics, visual observations.

\section{Introduction}

Visual meteor observation is an easy to grasp method that provides scientific outputs. After a couple of minutes spent by observations you can become a part of an international team, and you will be able to learn how the interplanetary matter surrounding the Earth is distributed. The principles are simple and provide an interesting and engaging alternative to practical exercises in physics. The requisites are: a sleeping bag, a camping mat, a flashlight, a stellar map with stellar magnitudes, and studying the following lines.

Visual meteor observations have a long tradition in Slovakia. The first national meteor expedition took place on 10.-25.7.1958 on the hill Bezovec which lies in Považský Inovec mountain range near Piešt’any. Since then, several observation groups have been formed in Slovakia. In 1998, the International Meteor Organization (IMO) was established. Its task is to coordinate scientific research

Jozef Drga, Alexander Dubček University of Trenčín, Trenčín, Slovakia; jozef.drga@tnuni.sk

Martin Bulko, Faculty of Mathematics, Physics and Informatics, Comenius University in Bratislava, Bratislava, Slovakia; jadrovystudent@gmail.com

Karol Petrík, Observatory and Planetarium in Hlohovec, Hlohovec, Slovakia; kpetrik@astronyx.sk

Mária Csatáryová, Faculty of Education, University of Prešov, Prešov, Slovakia; maria.csataryova@unipo.sk

Stanislav Šimkovič, High School of Piarists, Trenčín, Slovakia; s.simkovi@piaristi.sk 


\section{Acta Technologica Dubnicae \\ volume 6, 2016, issue 1}

activities in the field of meteor astronomy, and coordinate the collaboration between amateur astronomical observers and professional scientists. Because of recent technical advancements, high sensitive CCD and CMOS detectors are now picking up the baton of visual observations. However, the visual observations still play a role in determining the so called population index. Meteors can also be observed by radars or radio waves. This paper focuses on the description of methods dealing with obtaining and evaluating of results from visual meteor observations carried out by students, teachers and general public. A possibility of using these observations and evaluation of results as an alternative form of practical exercises in physics is also presented in this paper. Included is also the analysis of Lyrid meteor shower observations from the years 2009-2015.

\section{A few notes on meteor showers}

The term meteor shower refers to the increased frequency of meteor occurrence during a certain period of time. The trajectories of the meteors are approximately the same, and originate from a seemingly small area which can be considered to be a single point. This point is called the radiant. A meteor shower originates from comets releasing a material during the period of increased comet activity (usually when the comet is near its perihelion). Since meteor trajectories are roughly parallel, from Earth-meteors geometry it follows that a meteor does not start to glow directly in the radiant (see Figure 1). Beginners could pick up a suitable meteor shower for their observations by referring to Table 1 . The table shows the start and the end of meteor shower activity, presumed date of its maximum, the location of the radiant at the time when the maximum occurs in right ascension $(\alpha)$ and declination $(\delta)$ coordinates, average Zenithal Hourly Rate (ZHR), average value of the population index (r), and the code of the shower. A more complete list of meteor showers can be found on the IMO website (www.imo.net), or on the website of the International Astronomical Union. 
Table 1

Important meteor showers

\begin{tabular}{|c|c|c|c|c|c|c|c|c|}
\hline Name & 峦 & 롬 & 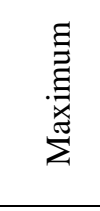 & 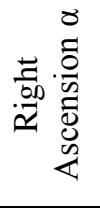 & 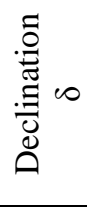 & 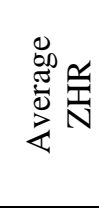 & - & $\frac{\pi}{8}$ \\
\hline Quadrantids & 28.12 & 12.1 & 4.1 & 230 & 49 & 100 & 2,1 & QUA \\
\hline Lyrids & 16.4 & 25.4 & 21.4 & 271 & 34 & 15 & 2,1 & LYR \\
\hline$\eta$ Aquariids & 19.4. & 28.5 & 5.5 & 338 & -1 & 40 & 2.4 & ETA \\
\hline$\delta$ Southern Aquariids & 12.7 & 24.8 & 30.7 & 240 & -16 & 15 & 3,2 & SDA \\
\hline August Perseids & 17.7 & 24.8 & 12.8 & 48 & 58 & 60 & 2,2 & PER \\
\hline к Cygnids & 3.8 & 25.8 & 18.8. & 286 & 59 & 3 & 3,0 & $\mathrm{KCG}$ \\
\hline$\alpha$ Aurigids & 28.8 & 5.9 & 1.9 & 91 & 39 & 7 & 2,5 & AUR \\
\hline September Perseids & 5.9 & 21.9 & 8.9 & 48 & 40 & 5 & 3,0 & SPE \\
\hline Draconids & 6.10 & 10.10 & 9.10 & 262 & 54 & $?$ & 2,6 & $\begin{array}{l}\text { GIA/ } \\
\text { DRA }\end{array}$ \\
\hline Orionids & 2.10 & 7.11 & 22.10 & 95 & 16 & 20 & 2,5 & ORI \\
\hline Northern Taurids & 10.9 & 20.11 & 11.10 & 32 & 9 & 5 & 2,3 & STA \\
\hline Southern Taurids & 20.10 & 10.12 & 12.11 & 58 & 22 & 5 & 2,3 & NTA \\
\hline Leonids & 6.11 & 30.11 & 17.11 & 152 & 22 & $15-?$ & 2,3 & LEO \\
\hline Geminids & 4.12 & 17.12 & 14.12 & 112 & 33 & 100 & 2,5 & GEM \\
\hline Ursids & 17.12 & 26.12 & 22.12 & 217 & 76 & 7 & 3,0 & URS \\
\hline
\end{tabular}

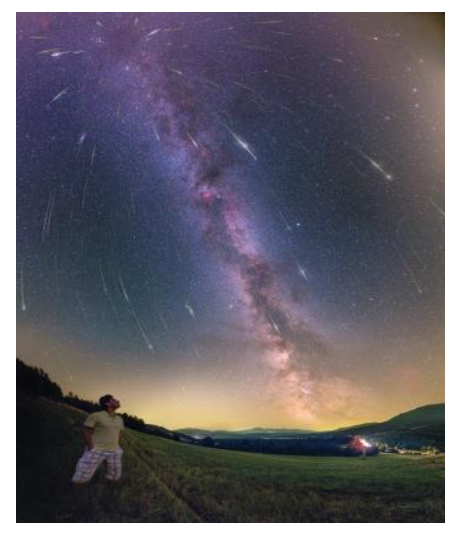

Figure 1. The photo of a meteor shower. 


\section{General information regarding the organization of observations}

The visual observation is usually carried out in the groups consisting of 2 to 6 observers. Each group also contains a person who records the observation results. The observers usually divide the sky either into quadrants based on the four cardinal directions plus the radiant, or they pick up the center of the visual field. Another good choice is the conic section of the sky with the apex in the location of the observer, angled at $30^{\circ}$ from the radiant. It is advisable to supplement the observation with some kind of group activity (a night game, barbecue, telescope observations). The counting method (also called C method) requires noting the time of meteor occurrence, the name of the meteor shower and the magnitude of the meteor.

An alternative method is the $\mathrm{P}$ (plotting) method. This method requires detailed knowledge of celestial sphere and is therefore suitable for advanced observers only. The name of the meteor shower where an observed meteor belongs to is determined by the direction and trajectory length of the meteor (the trajectory of the meteor extended backward must intersect the radiant). The closer to the radiant, the shorter is the meteor trajectory, as can be seen in Figure 1. A common scenario is that the trajectory of the meteor corresponds to the radiant, but in the vicinity of the radiant this trajectory is excessively long. In such a case the meteor does not belong to the meteor shower in question, and is part of the background. These background meteors, also called sporadic meteors, are not associated with any particular meteor shower.

The brightness (star magnitude) of the meteor is recorded with 0.5 magnitude accuracy. The brightness is determined from the comparison of the brightness of the meteor and a chosen star or the object with known brightness (Polaris -2 mag, Altair - 1 mag, Vega - 0 mag, Sirius - 1.5 mag, Jupiter - 2 mag, full Moon - $12 \mathrm{mag}$ ). Information about star brightness can be found in celestial atlases or celestial maps.

Sometimes it could be interesting to keep an eye on so called tracks in the shower. These tracks are caused by the gases ionized by the meteor, or by the subsequent recombination of ions. The duration of these tracks ranges from tenths of second to minutes.

It is important to determine the ability of an observer to discern poorly visible objects on the sky. This is related to the quantity called limiting magnitude. The limiting magnitude is defined as being the magnitude of the faintest star near the zenith that the observer can detect using the slightly averted naked eye. The method consists of counting the stars in triangular areas predefined by IMO 


\section{Acta Technologica Dubnicae \\ volume 6, 2016, issue 1}

(IMO, 2013a). Some of these areas are shown in Figure 2. The sensitivity of an observer's eye is determined according to the number of counted stars. For these purposes, the standard limiting magnitude is set to 6.5 magnitudes.

The observation should be divided into intervals. The recommended duration of an interval is $0.5-1.5 \mathrm{~h}$. The breaks start at the beginning of the first minute and finish at the end of the last minute of the break. No meteor must be recorded for the observer taking the break. The duration of the breaks is recorded in order to determine the effective observing time.

The observation must meet the following criteria:

a) The radiant must be higher than $20^{\circ}$ above the theoretical horizon.

b) The value of observer's limiting magnitude must be at least equal to 5 magnitudes.

c) The part of the sky assigned to an observer must not be covered by clouds or ground objects by more than $20 \%$.

d) The observation cannot be realized if the Moon is full, or nearly full.

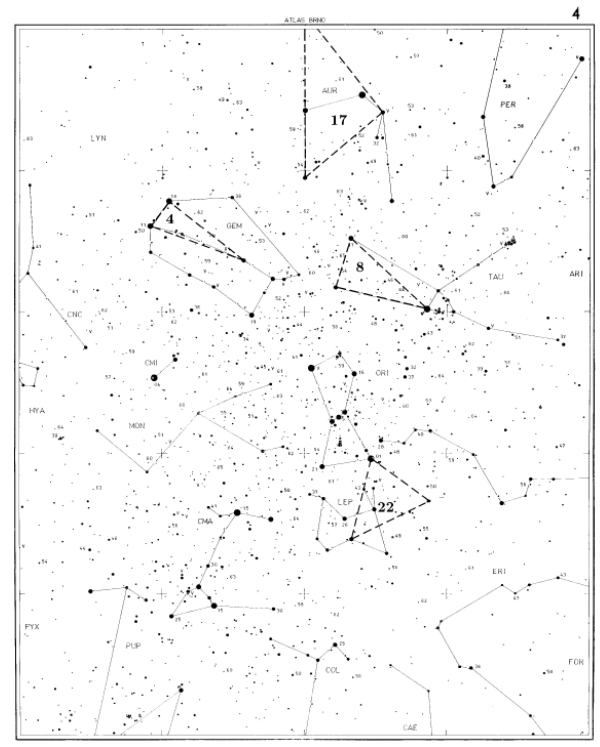

Figure 2. The areas at the celestial map used for determining the limiting magnitude $(4,8,17,22)$. 


\section{Mathematical background}

From the observation it is possible to determine the so called Zenithal Hourly Rate (ZHR) which is defined as:

$$
\mathrm{ZHR}=\frac{\mathrm{N} \mathrm{F} r^{6.5-M}}{\mathrm{~T}_{\text {eff }} \sin (\mathrm{h})}
$$

$\mathrm{N}$ is the number of observed meteors, $\mathrm{F}$ is the correction factor due to obstruction of the field of view, $T_{\text {eff }}$ is the effective time of observation, $M$ is the limiting magnitude and $\mathrm{h}$ is the radiant altitude. The field of view correction factor $F$ is given by:

$$
F=\frac{1}{1-\mathrm{k}}
$$

$\mathrm{k}$ is the percentage of the observer's field of view which is obstructed (by clouds, for example). For instance, if the average obstruction during the observation was $10 \%$, then $\mathrm{k}=0.1$. The effective observing time is defined as:

$$
\mathrm{T}_{\text {eff }}=T-\sum_{1}^{A} B_{i}
$$

$\mathrm{B}_{\mathrm{i}}$ is the duration of a break, $\mathrm{A}$ is the number of breaks and $\mathrm{T}$ is the total interval length. Lastly, the population index is given by:

$$
\mathrm{r}=\frac{\mathrm{N}(\mathrm{m}+1)}{\mathrm{N}(\mathrm{m})}
$$

$\mathrm{N}(\mathrm{m}+1)$ is the number of meteors of magnitude $\mathrm{m}+1$ and $\mathrm{N}(\mathrm{m})$ is the number of meteors of magnitude $\mathrm{m}$. From these observations it is possible to determine the spatial particle density and the mass index (Koschack, Rendtel, 1988; Koschack, Rendtel, 1990a; Koschack, Rendtel, 1990b).

All these calculations will be carried out by the International Meteor Organization, you do not have to deal with them in detail.

\section{Electronic visual report form}

The forms below validate and submit data for entry in the IMO Visual Meteor Database and the creation of ZHR activity graphs (IMO, 2013b). In the following paragraphs we will provide information how to fill in all these forms. 


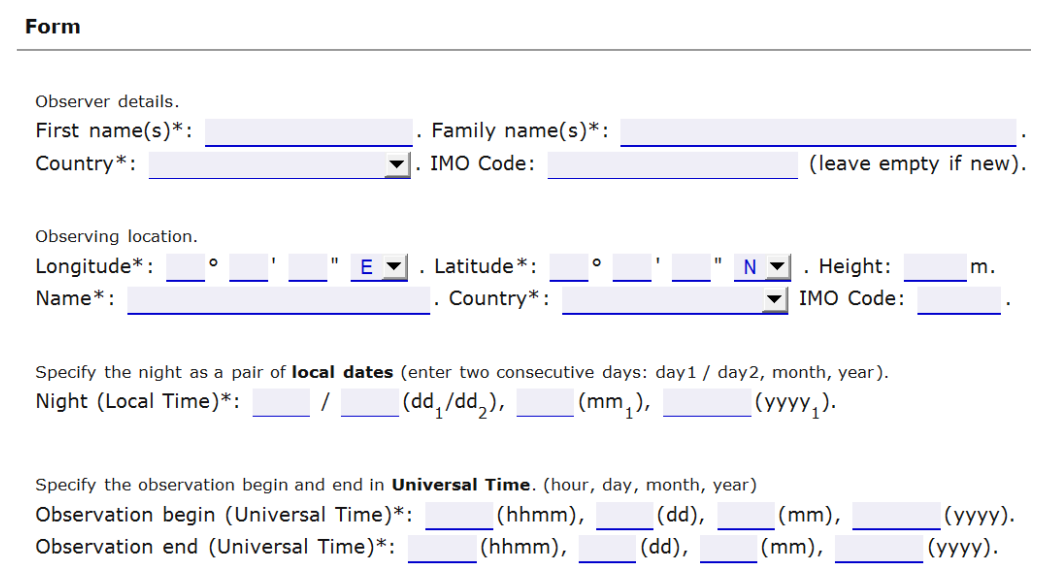

Figure 3. Visual report form Part 1 - Basic information.

The first section requires entering some of the observer's personal data - First name, Family name, Country and IMO Code (if it has been assigned). The section named Observing location contains fields for entering longitude, latitude, height (above sea level), name of the observation place, and country and IMO code of the observation place (for instance, Trenčín IMO code is 23711). The next section requires specifying the night of observation as a pair of local dates (two consecutive days should be entered). Lastly, one needs to specify the beginning and the end of the observation in Universal Time (UT). The conversions between UT, Central European Time (CET) and Central European Summer Time (CEST) are as follows:

$\mathrm{UT}=\mathrm{CET}-1$ hour

$\mathrm{UT}=\mathrm{CEST}-2$ hours

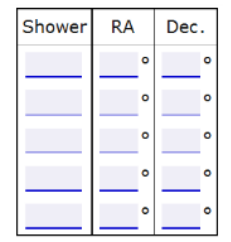

Figure 4. Visual report form Part 2 - Showers considered for observation

In "Part 2 - Showers considered for observation" we have to insert the IMO three-letter codes of the observed meteor showers. The codes can be found in (Meteor showers, 2014). "RA" stands for right ascension and "Dec." for declination on the day of observation. Note that if the meteor shower is included in the IMO list, right ascension and declination will be calculated automatically. 


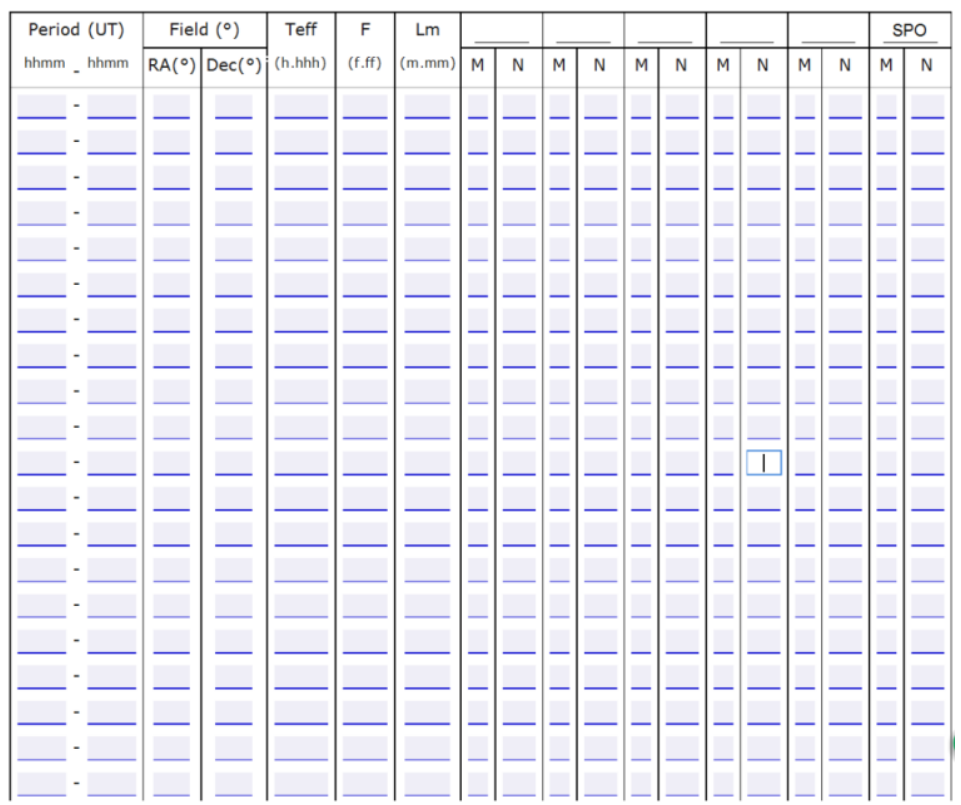

Figure 5. Visual report form Part 3 - Observed number of meteors per period and per shower.

Part 3 of the Visual report form shown in Figure 5 requires entering the beginning and the end of individual observation intervals. In case of shower maxima or outbursts, short observation periods should be used (e.g. 5 minutes). As a rule of thumb, one row (one time interval) should contain no more than 20 observed meteors. The table also contains fields for entering the right ascension (RA), declination (Dec), effective observation time (Teff), field of view correction factor $(\mathrm{F})$, and limiting magnitude $(\mathrm{Lm})$. The columns marked as $\mathrm{M}$ or $\mathrm{N}$ are for entering the method of observation and the number of observed meteors, respectively. The last two columns are reserved for sporadic meteors. 


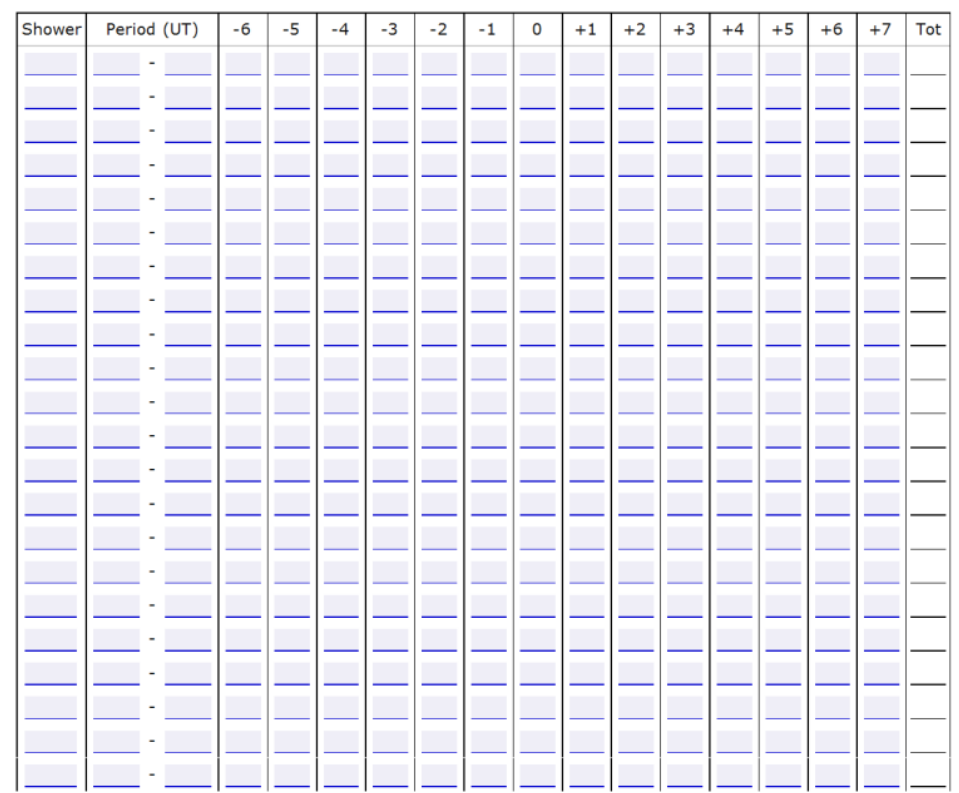

Figure 6. Visual report form Part 4 - Magnitude distributions for showers and sporadic meteors.

Part 4 of the Visual report form shown in Figure 6 is important for determining the population index (formula 4). The column "Shower" should contain the codes of individual meteor showers. It is recommended to use the same time interval as in Part 3. It is also possible to merge the intervals into bigger ones, but one row should not contain more than 30 meteors.

The rest of the columns in the table are marked from -6 to 7, and are incremented by 1 . The observer is supposed to enter here the number of observed meteors of a given magnitude. For instance, if we detected two meteors of the magnitude 3, then we should enter the number two in the column " 3 ". If we detected a meteor of the magnitude 1.5 , we should enter the number 0.5 in the columns " 1 " and " 2 ", respectively. The column 'Tot' (total) will be calculated automatically. Also, please do not forget to include the magnitude distribution of sporadic meteors (SPO). 


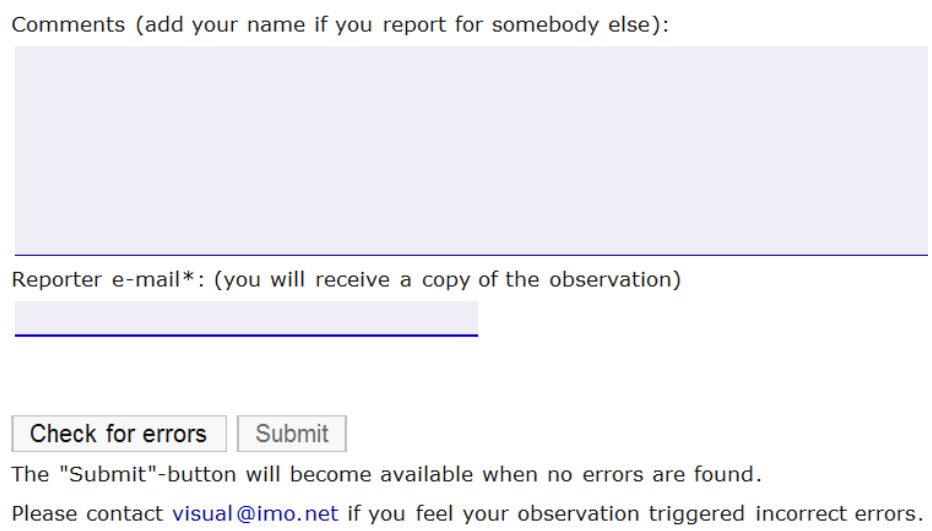

Figure 7. Visual report form Part 5 - Comments \& email.

The last part of Visual report form (Figure 7) is reserved for comments and the e-mail of the person who filled in the Visual report form (the reporter). Comments field can be used to enter the name of the reporter, description of observation conditions etc. Lastly, we click on "Check for errors" button and if no errors are found we can submit the whole report by clicking on "Submit".

\section{The Lyrid meteor shower}

As a practical example, the ZHR curve of the Lyrid meteor shower is evaluated in this section. The Lyrid meteor shower is active from April 16 to April 26 (McBeth, 2014). The maximum usually occurs on April 22. This meteor shower is related to C/1861 G1 Thatcher comet (Molina \& Moreno, 2013).

In order to increase the sample size and reduce the statistical error, the ZHR data from 7 consecutive years (2009-2015) were used. In total, 4910 Lyrid meteors and 1746 time intervals were taken into account. The population index value of 2.1 was used.

The Lyrid meteor shower has been studied by various techniques (Porubcan, Hajduk, Cevolani, \& Trivellone, 1996; Porubcan \& Simek, 1988; van Wassenhove, 1987). According to our analysis based on fitting the ZHR profile by a suitable function, the meteor shower activity may actually start sooner (April 11) and finish later (May 3) (Figure 8). The following Gaussian-parabolic function was used for fitting (Drga \& Janek, 2014; Drga \& Bulko, 2014):

$$
\mathrm{ZHR}=\mathrm{A}(\mathrm{x}-\mathrm{B})^{2}+\mathrm{C}+\mathrm{De}^{-\frac{1}{2}\left(\frac{\mathrm{x}-\mu}{\sigma}\right)^{2}}
$$

The fitting parameters are shown in Table 2 . 


\section{Table 2}

Fitting results.

\begin{tabular}{ccc}
\hline Parameter & Value & Error \\
\hline A & $-0,025$ & 0,009 \\
B & 31,5 & 1,3 \\
C & 3,7 & 0,3 \\
D & 11,4 & 0,7 \\
$\sigma$ & 0,49 & 0,05 \\
$\mu$ & 32,23 & 0,04 \\
\hline
\end{tabular}

The position of the peak of meteor shower activity (this value can vary) was found at solar longitude $\lambda=32.21$, which is in approximate agreement with the value of 32.32 published in (McBeth, 2014). The difference between these values is ca. 2.64 hours.

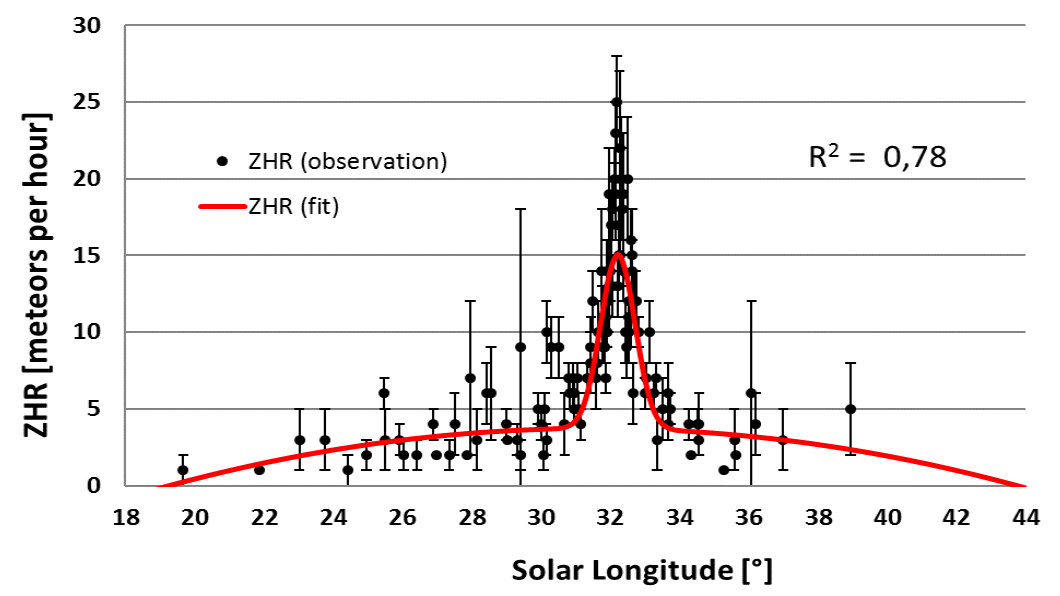

Figure 8. ZHR profile of the Lyrid meteor shower.

\section{Conclusion}

Visual meteor observations can be used as a fun addition to the laboratory exercises in physics. They are suitable for lower and upper secondary education, as well as for freshmen at universities and general public. There are many possible outputs of these observations, i.e. the graph of ZHR as a function of time, ZHR as a function of the observer, limiting magnitude as a function of time, or limiting magnitude as a function of the observer. Population index and 


\section{Acta Technologica Dubnicae \\ volume 6, 2016, issue 1}

other relevant parameters can also be computed if we want to deal with this subject in more detail. The authors of this paper hope that the teachers will find this contribution useful and that it will make the teaching and learning physics more appealing. As a practical example, the ZHR profile of the Lyrid meteor shower was analyzed by a combination of Gaussian and parabolic function.

\section{References}

Drga, J., \& Bulko, M. (2014). Determination of average maximum of Orionids meteor shower from visual observations. University review, 8(3-4), 104107.

Drga, J., \& Janek, M. (2014). Determination of the maximum of the Geminid meteor shower from visual observations. WGN Journal of the International Meteor Organization, 42(3), 125-126.

IMO. (2013a). Determination of the limiting magnitude. Retrieved from http://www.imo.net/visual/major/observation/lm

IMO. (2013b). Electronic visual report form. Retrieved from http://www.imo.net/visual/report/electronic

Koschack, R., \& Rendtel, J. (1988). Number density in meteor streams. WGN Journal of the IMO, 16(5), 149-157.

Koschack, R., \& Rendtel, J. (1990a). Determination of spatial number density and mass index from visual meteor observations (I). WGN - Journal of the IMO, 18(2), 44-58.

Koschack, R., \& Rendtel, J. (1990b). Determination of spatial number density and mass index from visual meteor observations (II). WGN - Journal of the IMO, 18(4), 119-140.

McBeth, A. (2014). IMO meteor shower calendar 2014.

Meteor showers: IAU 3-letter codes (2014). Retrieved from http://star.arm.ac.uk/ dja/shower/codes.html

Molina, A., \& Moreno, F. (2013). Lyrids and perseids meteoroids: reconciliation and discrepancy between cometary outgassing theory and electrophonic sound data. The astronomical journal, 145(4), 4.

Porubcan, V., Hajduk, A., Cevolani, G., \& Trivellone, G. (1996). Lyrid meteor shower observed by a forward scatter meteor radar, Contributions of the astronomical observatory Skalnate Pleso, 26, 5-9.

Porubcan, V., \& Simek, M. (1988). Distribution of Lyrid meteoroids in a large range of echodurations. Bulletin of Astronomical institutes of Czechoslovakia, 39, 165-168.

Van Wassenhove, J. (1987). Belgian and Danish radio observations in April 1987. WGN Journal of the International Meteor Organization, 16(4), 123126. 